\title{
On stability of nonlinear atomic vibrations in strained carbon chains
}

\author{
G.M. Chechin ${ }^{\dagger}$, D.A. Sizintsev, O.A. Usoltsev \\ †gchechin@gmail.com
}

Southern Federal University, Department of physics, Zorge Str., 5, 344090, Rostov-on-Don, Russia

\begin{abstract}
Monoatomic carbon chain (carbyne) can exist in two different modifications: cumulene with double chemical bonds between its atoms, and polyyne with alternation of single and triple bonds. In our previous paper [Letters on materials 6 (2), 146 - 151 (2016)], we have discussed a new physical phenomenon which was revealed in cumulene chains under uniform strain. It was found that above $11.2 \%$ strain softening of $\pi$-mode atomic vibrations takes place in some range of its amplitude. Condensation («freezing») of this soft mode leads to structural phase transition of displacement type. It is the Peierls phase transition which was found earlier in [Nano Lett. 14, 4224-4229 (2014)] with the aid of a different approach. The above phenomenon occurs due to the fact that old atomic equilibrium positions (EQPs), near which atoms vibrate in the case of small strain, lose their stability and two new EQPs appear near each of them. The $\pi$-mode softening corresponds to vibrations in the vicinity of these new EQPs. In the present paper we discuss the problem of stability of the new EQPs, as well as a possibility of condensation in cumulene of two other symmetry-determined nonlinear normal modes, different from the $\pi$-mode. Inferences of this paper may be useful for comparison of the results, obtained by methods of molecular dynamics, with those obtained with the aid of $a b$ initio simulations based on the density functional theory in studying different physical phenomena.
\end{abstract}

Keywords: carbon chains, nonlinear normal modes, anharmonic vibrations, ab initio calculations, phase transitions.

\section{Об устойчивости нелинейных атомных колебаний в растянутых углеродных цепочках}

\author{
Чечин Г.М. ${ }^{\dagger}$, Сизинцев Д.А., Усольцев О.А. \\ †gchechin@gmail.com
}

Южный Федеральный Университет, ул. Зорге, 5, 344090, Ростов-на-Дону, Россия

\begin{abstract}
Моноатомная углеродная цепочка (карбин) может существовать в двух различных модификациях: кумулен, который характеризуется двойными химическими связями между атомами, и полиин, в котором имеет место чередование одинарных и тройных связей. В предыдущей нашей статье [Letters on materials 6 (2), 146-151 (2016)] было обнаружено новое физическое явление в цепочках кумулена, подвергнутых однородному растяжению. Оказалось, что при растяжениях выше 11.2\% наблюдается смягчение атомных колебаний, описываемых $\pi$-модой в некотором интервале её амплитуд. Конденсация («замерзание») этой мягкой моды приводит к структурному фазовому переходу типа смещения. Этот переход является фазовым переходом Пайерлса, который был обнаружен ранее в работе [Nano Lett. 14, 4224 - 4229 (2014)], но в рамках отличного от использованного нами подхода. Вышеуказанное явление обусловлено потерей устойчивости старых положений равновесия (EQPs), относительно которых атомы углерода совершают колебания при малых растяжениях, и возникновением около каждого из них двух новых EQPs. Cмягчение $\pi$-моды соответствует колебаниям атомов углерода в окрестности этих новых EQPs. В настоящей работе исследуется проблема устойчивости новых положений равновесия, а также возможность конденсации в кумулене двух других симметрийно-обусловленных нелинейных нормальных мод, отличных от $\pi$-моды. Результаты данной работы могут оказаться полезными для сравнения расчётов, проведенных с помощью методов молекулярной динамики, с первопринципными расчётами, выполнеными в рамках теории функционала плотности, при исследовании различных физических явлений.
\end{abstract}

Ключевые слова: углеродные цепочки, нелинейные нормальные моды, негармонические колебания, первопринципные расчёты, фазовые переходы. 


\section{Introduction}

Carbyne represents a chain of carbon atoms and can exist in two different forms. Cumulene, whose chemical formula is $(=\mathrm{C}=\mathrm{C}=)_{\mathrm{n}}$, represents more symmetrical structure with double bonds between all its atoms, while polyyne with chemical formula $(-\mathrm{C} \equiv \mathrm{C}-)_{\mathrm{n}}$ represents carbon chain with alternating single and triple bonds. There is one carbon atom in the unit cell of cumulene and there are two atoms in the unit cell of polyyne.

Because of many unique mechanical, physical and chemical properties carbyne is considered as perspective material for various nanodevices, for hydrogen storage, etc. (see [1-5] and papers cited therein).

Chemical synthesis of pure carbyne chains and their experimental study are very difficult and, therefore, theoretical $a b$ initio investigations, in particular those based on the density functional theory (DFT), play a rather important role for prediction of its properties and for treating different physical phenomena, which are possible in this material. DFT computer simulations lead to many interesting results on carbyne chains. Especially we would like to refer to the papers [1 -4] of Rice University group in Houston (USA) and to the paper [5], which are close to the subject of our own investigation.

During the study of different types of nonlinear atomic vibrations in strained cumulene chains, we revealed an unexpected phenomenon of softening of the longitudinal $\pi$-mode vibrations above some critical value of the strain. Results of this study were partially published in the paper [6]. They can be summarized as follows.

For strains lower than $\eta=11 \%$ cumulene demonstrates monotonic hard type of nonlinearity (the frequency increases with increasing the $\pi$-mode amplitude $a$ ). However, for $\eta>11.2 \%$ there is a certain range of amplitudes $a$ in which soft nonlinearity occurs, namely, the frequency of the $\pi$-mode abruptly decreases and then again begins to increase.

The phenomenon of vibrational modes softening is well known in the theory of structural phase transitions [7] where by condensation ("freezing") of such modes one tries to explain the nature of the displacement-type phase transitions. This is the so-called concept of soft modes. It is essential that in the majority of the papers on this subject, soft modes are treated in purely phenomenological manner with some vague arguments about changing of electronphonon interactions in crystal under change of such external parameters as temperature and pressure. Unlike these works, in our study a soft vibrational mode in cumulene appears as a direct result of the ab initio simulation without any additional assumptions.

The condensation of the $\pi$-mode induces the Peierls phase transition associated with doubling of the unit cell of cumulene and, as a consequence, this material transforms into the polyyne form of carbyne. The physical nature of this phenomenon can be explained by the stability loss of the old equilibrium positions (EQPs) of the carbon atoms and appearance of two new equilibrium positions near each of them.

In the excellent paper [1], the structural transformation of cumulene under certain strain was revealed two years before the publication of our paper also by DFTmethods, but in the framework of another approach. Comparison of our results with those from [1] will be done bellow ${ }^{1}$.

We were able to explain the nature of $\pi$-mode softening by considering potential energy profiles obtained by ab initio DFT-simulations. Moreover, we have introduced a simple classical model, which explains all observed phenomena in cumulene under strain. This model represents a chain of mass points whose interactions are described by the Lennard-Jones potential (L-J chain). Such model allows one to understand the cause of appearance of new equilibrium positions near old ones when cumulene undergoes an appropriate strain.

Note that in [1] another simple model was introduced to explain the properties of the Peierls phase transition. The authors of this paper considered quantum-mechanical motion of the atom moving in a double-well potential constructed by DFT-simulations. They studied the evolution of several lowest energy levels in this potential with changing of its form under increase of the cumulene strain.

In our interpretation, as well as in the software package ABINIT $[8,9]$, which we used for our simulations, nuclear motion is treated by classical mechanics, while quantum approach is used only for electron subsystem (Kohn-Sham equations [10] are solved for every change of nuclear configuration).

The main ab initio results obtained in our work and in the paper [1] are sufficiently close to each other. Some discrepancy can be explained by different approximations used in the framework of DFT-approach (different exchangecorrelation functionals, different sets of basis functions for solving KohnSham equations, different realization of the numerical methods in packages ABINIT and VASP, etc.). Nevertheless, our results and those from [1] are fully identical qualitatively (detailed comparison will be presented elsewhere).

Importance of studying Peierls phase transition in the carbon chains is connected with the radical change of carbyne electron spectrum induced by this transformation. Indeed, it leads to appearing of an energy gap in this spectrum and, as a consequence, the conductive cumulene transforms into polyyne that becomes an insulator or semiconductor. In turn, this phenomenon opens perspectives to control electrical behavior of carbyne by mechanical strain [1].

In the present paper, we study the stability of the abovediscussed new equilibrium positions, both in the framework of $a b$ initio approach and the simple LennardJones model. In this way, we have revealed some unexpected properties. We feel that it is essential to keep them in mind for any comparison of ab initio results with those obtained by the methods of molecular dynamics.

With the aid of our approach combined with some group-theoretical methods [11] we predict the possibility of existence of two new types of carbon chains, besides cumulene and polyyne. They both possess alternation of bond lengths (BLA), but with different alternating schemes compared to that of the polyyne.

1 Unfortunately, we were not aware of the paper [1] when prepared our own paper [6]. 


\section{Mathematical models and numerical experiments}

We consider longitudinal nonlinear atomic vibrations in the uniformly strained chain. This strain is modeled by an artificial increase of the unit cell size $(R)$ with respect to that of the chain without strain $\left(R_{0}\right)$. Speaking about the strain of the chain by $\eta$ per cent, we mean that $R=R_{0}(1+\eta)$.

In the present paper, we use two different mathematical models for studying atomic vibrations, "DFT model" and "L-J model". In both models, the periodic boundary conditions are assumed.

In the DFT model, the quantum-mechanical approach is used to describe interatomic interactions in the framework of the density functional theory [10]. For the computer simulations, we have used the software package ABINIT [ 8 - 9]. Some approximations are made for studying atomic dynamics. The Born-Oppenheimer approach is used to separate the fast motion of electrons and slow motion of nuclei. At each time-step for fixed position of nuclei, self-consistent electron density distribution is calculated by solving Kohn-Sham quantum-mechanical equations. Then forces acting on the nuclei are computed, and their new configuration is found by performing one step of solution of the classical dynamical equations. For this new configuration, the procedure of selfconsistency for the electron subsystem is repeated.

All our calculations are performed in the framework of the local density approximation (LDA). Pseudo-potentials by Troullier-Martins are used to describe the field of the carbon atoms inner shells in the process of the Kohn-Sham equations solving with the aid of the plane waves basis (with energy cutoff equal to $1360 \mathrm{eV}$ ). The convergence for energy is chosen as $10^{-8} \mathrm{eV}$ between two steps.

The second our model is a conventional one in the framework of the classical molecular dynamics. The main idea of this approach can be formulated as follows. Molecules (atoms) are replaced by mass points whose interactions are described with the aid of some phenomenological potentials. For the obtained dynamical system, equations of classical mechanics are solved. In the framework of quantum mechanics, such approach cannot be considered as adequate, because it is difficult or impossible to find potentials, which are good enough to take into account the influence of atomic electron shells on dynamical properties of the original physical system. That is why one has to use very complicated many-particle potentials, which possess different forms for different geometry of the interacting atoms and contain phenomenological constants defined by huge tables.

However, in some cases, reasonable results can be obtained even with the aid of simple pair potentials, such as those by Morse, Lennard-Jones, etc. Bellow, we try to explain results, obtained by ab initio calculations for the $\pi$-mode dynamics in carbyne, using the model of a chain whose particles interact via the Lennard-Jones potential.

All our numerical experiments in the framework of the L-J model were performed with the aid of the Runge-Kutta method realized in the software package MAPLE- 11 .

\section{3. $\pi$-mode vibrations and structural atomic transformation in strained carbon chains}

In this section, we reproduce some results from [6], which are necessary for our further discussion.

1) Above a certain critical value of the strain, old equilibrium positions of all carbon atoms lose their stability and two new EQPs appear near each of the old ones. In turn, this phenomenon results in softening of $\pi$-mode in some range of its amplitude because atoms begin to vibrate about new EQPs.

2) Condensation of the $\pi$-mode leads to a new atomic equilibrium configuration that corresponds to the Peierls phase transition. After this transition, the unit cell turns out to be twice as large than that of cumulene, and the carbon chain transforms into another carbyne form, polyyne, with bond lengths alternation. As was already mentioned, this phenomenon is of great physical importance, because such atomic transformation leads to qualitative change in carbyne electron spectrum.

3) The clear interpretation of the above phenomenon of appearing of new equilibrium positions can be given with the aid of the simple classical model representing the mass-point chain whose interparticle interactions are described by the Lennard-Jones potential.

\section{Study of stability of the carbyne new equilibrium atomic positions in the framework of the Lennard-Jones model}

In Fig. 1, we present potential energy profile corresponding to the $\pi$-mode vibrations in one unit cell of cumulene under the strain $\eta=15 \%$ (this profile was obtained in [6] with the aid of DFT simulation).

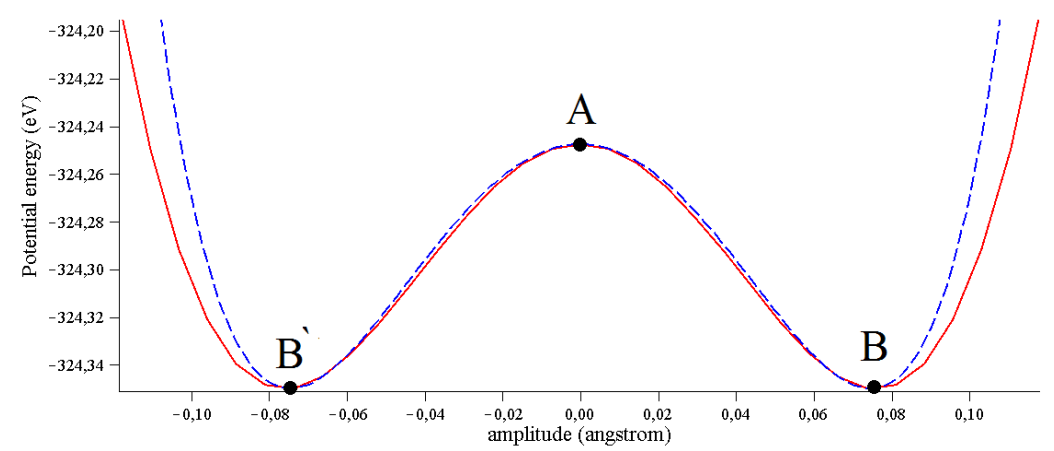

Fig. 1. The points A and B (B') correspond to the old and new equilibrium positions of carbon atom, respectively. The solid curve shows the $a b$ initio results, while the appropriate fitting in the framework of the Lennard-Jones model is shown by the dashed curve. 
The set of atomic displacements $\boldsymbol{X}(t)=\left[x_{1}(t), x_{2}(t), \ldots, x_{N}(t)\right]$ of the $N$-particle carbon chain for the $\pi$-mode vibrational regime at a fixed time $t=t_{0}$ can be written as follows:

$$
\boldsymbol{X}\left(t_{0}\right)=\left[a\left(t_{0}\right),-a\left(t_{0}\right)\left|a\left(t_{0}\right),-a\left(t_{0}\right)\right| \ldots \mid a\left(t_{0}\right),-a\left(t_{0}\right)\right] .
$$

In this pattern, all $x_{i}(t)$ with odd numbers are equal to $a\left(t_{0}\right)$, while those with even numbers are equal to $-a\left(t_{0}\right)$. Thus, all neighboring atoms vibrate out of phase with equal amplitudes as shown in Fig. 2.

From this figure, one can see that at the given instant $\left(t_{0}\right)$ the following alternation of the atomic distances occurs:

$$
B L A_{\pi}=\left[l_{1}, l_{2}\left|l_{1}, l_{2}\right| \ldots \mid l_{1}, l_{2}\right] .
$$

Here $l_{1}=l_{0}-a, l_{2}=l_{0}+a$, while $l_{0}$ is the equilibrium distance between atoms in the strained cumulene and $a=a\left(t_{0}\right)$. This BLA corresponds to the polyyne form of carbyne.

If we consider all atoms at new EQPs, i.e. at the distance $a_{0}$ from the old EQPs, then the bond lengths in Eq. 2 are equal to $l_{1}=l_{0}-a_{0}, l_{2}=l_{0}+a_{0}$, and the difference $\Delta l$ of these bonds is

$$
\Delta l=2 a_{0} .
$$

Certainly, the parameter $a_{0}$ depends on the value of the strain $\eta$ and this dependence $a_{0}(\eta)$ is presented in Fig. 3.

In Fig. 1, we depict by the dashed line the energy profile obtained for the L-J chain by a certain fitting [6]. The LennardJones potential is determined by two phenomenological parameters which can be found from the condition of its coincidence with the $a b$ initio profile at two points, A and B. Fig. 1 corresponds to the strain $\eta=15 \%$ and for this case is equal to $0.075 \AA$.

Appearance of new equilibrium positions can be easily explained in the framework of the L-J chain model [6]. Indeed, they appear even in the simplest case of two fixed atoms (say, \#1 and \#3) with another atom (\#2), which may be displaced at different distance from its EQP at the middle of

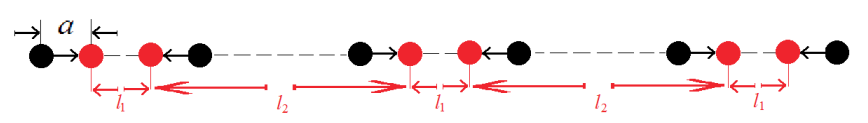

Fig. 2. Bond lengths alternation in strained carbyne for the pattern (1) with $a\left(t_{0}\right)=a$. Here, for $a>0, l_{1}$ and $l_{2}$ are short and long bonds, respectively, and vice versa for $a<0$.

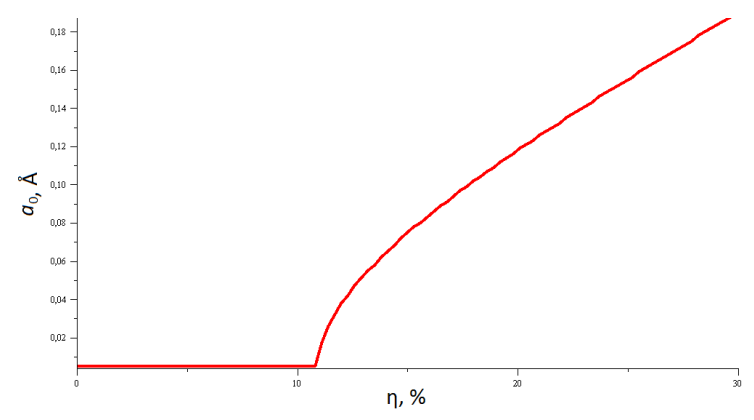

Fig. 3. Dependence of the $\pi$-mode parameter $a_{0}$ on the strain $\eta$ which corresponds to the minimum of potential energy (point B in Fig. 1). the atoms \# 1 and \#3. For finding any equilibrium position $(x)$ of the atom $\# 2$, we must equate by absolute value the forces $f_{l}(x)$ and $f_{r}(x)$ acting on it from the left and right neighboring atoms (these forces possess opposite directions). Then the equation $f_{l}(x)=f_{r}(x)$ for the forces, generated by the LennardJones potential, represents a nonlinear algebraic equation that can be solved by a simple graphic method. Intersections of the graphs of $f_{l}(x)$ and $f_{r}(x)$ turn out to be the equilibrium positions of the atom $\# 2$ between two fixed atoms, \#1 and \#3. In this way, we can visually find the cause of appearance of new equilibrium positions ${ }^{2}$.

In [6], we have solved the problem of existence of the new equilibrium positions for strained carbon chains in the framework of DFT ab initio approach, as well as for the Lennard-Jones model, while here we consider the problem of their stability. This problem can be formulated as follows. We must verify if a given new equilibrium position corresponds to a certain minimum of the potential energy $U(\boldsymbol{X})$ which is a function of $N$-dimensional vector $\boldsymbol{X}=\left[x_{1}, x_{2}, \ldots, x_{N}\right]$ of all degrees of freedom of our chain. Note, that the minimum in Fig. 1 corresponds to the one-dimensional function $u(a)$, where $a$ is the $\pi$-mode amplitude, and the vector $\boldsymbol{X}(t)=[a,-a|a,-a| \ldots \mid a,-a]$ determines the position of this minimum in the $\mathrm{N}$-dimensional space.

For example, below we study stability of the new equilibrium positions for the carbon chain of $N=8$ atoms.

Let us again consider the point $B$ at the bottom of the right potential well in Fig. 1. If $B$ represents a minimum of the potential energy $U(\boldsymbol{X})$ in the full eight-dimensional space of all possible displacements, then infinitesimal shift from this point in any direction leads to increase of the function $U(\boldsymbol{X})$.

It is well known that the local extremum of the function $U(\boldsymbol{X})$ in many-dimensional space can be of different type (minimum, maximum or a saddle point). The most effective way to analyze the type of a given extremum point $\boldsymbol{X}_{0}$ (it determines the equilibrium position) can be carried out for the L-J model by the following procedure.

Let us expand the function $U(\boldsymbol{X})$ near the point $\boldsymbol{X}_{0}$ into many-dimensional Taylor series and restrict it by quadratic terms only (this procedure corresponds to analyzing the harmonic approximation in the framework of dynamical approach). The obtained quadratic form is then transformed by a certain linear transformation of variables to the canonical form, which represents a superposition only of squares of new variables with some coefficients $\lambda_{j}(j=1 . . N)$. These coefficients can be found by diagonalization of the matrix of the original quadratic form as its eigenvalues. The extremum $\boldsymbol{X}_{0}$ will be a minimum only if $\lambda_{j} \geq 0$ for all $j=1 . . N$. If a certain eigenvalue turns out to be negative $\left(\lambda_{\mathrm{j} 0}<0\right)$, then any infinitesimal shift by $\gamma$ from the point $\boldsymbol{X}_{0}$ along the line $\boldsymbol{X}=\boldsymbol{X}_{0}+\gamma \boldsymbol{\xi}_{j 0}$, where $\xi_{j 0}$ is the eigenvector corresponding to $\lambda_{j 0}$, leads to decrease of the function $U(\boldsymbol{X})$ and, therefore, $\boldsymbol{X}_{0}$ is a saddle point (or maximum, if all $\lambda_{\mathrm{j}} \leq 0$ ).

With the aid of the above procedure, we have obtained the following results on stability of the new EQPs for the eightparticle L-J chain under uniform strain $\eta=15 \%: \lambda_{1}=0$, $\lambda_{2}=+88.4, \lambda_{3}=\lambda_{4}=+100.6, \lambda_{5}=+110.6, \lambda_{6}=\lambda_{7}=-12.2$,

2 The similar procedure can be also carried out for L-J chains with an arbitrary number of atoms. 
$\lambda_{8}=-22.2$ (these values were rounded up to 2 figures after decimal point). This result is disappointing since the new EQP turns out to be a saddle point (three $\lambda_{j}<0$ ) and, therefore, it is unstable.

To verify this sad result, obtained in the framework of the LJ model, we have found the energy profiles along the eigenvectors $\xi_{j}$ corresponding to all $\lambda_{j}(j=1 . .8)$. These vectors are listed in Table 1.

Let us comment on this table. All eigenvectors $\xi_{j}$ are orthogonal to each other and are linear independent. Therefore, their complete set can be used as a basis in eightdimensional space.

Note that these vectors can be obtained without studying of any dynamical model (in particular, without considering the L-J chain). One can find them with grouptheoretical methods only. Indeed, they are basis vectors of the irreducible representations (irreps) of the symmetry group $\mathrm{C}_{4 \mathrm{v}}$ constructed in the eightdimensional space of all atomic displacements of the chain. In the present paper, we will not consider this problem in detail, and restrict ourselves only with some comments on the group $\mathrm{C}_{4 v^{\circ}}$.

In Fig. 4, we present our eight-particle chain with periodic boundary conditions as a ring where arrows show the atomic displacements associated with the $\pi$-mode pattern $[a,-a|a,-a| a,-a \mid a,-a]$. For $a=0$ the diagram in Fig. 4 possesses point symmetry group $\mathrm{C}_{8 \mathrm{v}^{\circ}}$. This group contains 16 symmetry elements: 8 rotations around axis normal to the plane of the figure and passing through the center of the circle, as well as 8 mirror planes containing this axis. However, for the case $a \neq 0$ the symmetry of the pattern decreases by a factor two. Only eight elements of the above list survive: 4 rotations (by $\left.0^{\circ}, 90^{\circ}, 180^{\circ}, 270^{\circ}\right)$ and 4 mirror planes $\left(\sigma_{1}, \sigma_{2}\right.$, $\left.\sigma_{3}, \sigma_{4}\right)$. Therefore, the symmetry group of the displacement pattern in Fig. 4 is equal to $\mathrm{C}_{4 \mathrm{v}}$

On the other hand, this group possesses 4 one-dimensional irreps $(\Gamma 1, \Gamma 2, \Gamma 3, \Gamma 4)$ and 1 two-dimensional irrep (Г5). Namely, the basis vectors of these irreps are listed in Table 1. Note that doubly degenerate eigenvalues correspond to the twodimensional irrep.

Note that eigenvalues $\lambda_{j}$ from Table 1 turn out to be not important for our purpose (they depend on the value of the strain), but signs of $\lambda_{j}$ are important since they determine the type of the extremum $\boldsymbol{X}_{0}$ of the function $U(\boldsymbol{X})$.
Now we can analyze the energy profiles $U(\boldsymbol{X})$ along eight lines $\boldsymbol{X}=\boldsymbol{X}_{0}+\gamma \boldsymbol{\xi}_{j 0}(j=1 . .8)$ in eight-dimensional space. Here $\gamma$ is a sufficiently small number because we want to compare the corresponding results with those obtained for energy function considered as a quadratic form. The energy profiles $U(\boldsymbol{X})=u_{j}(\gamma)$ for all basis directions $\boldsymbol{\xi}_{j}$ are depicted in Fig. 5. We exclude the vector $\xi_{2}$ from our consideration because it determines the direction along the $\pi$-mode and, therefore, it has no relation to stability of this mode.

The graphs from this figure fully confirm the previously discussed results on stability properties of the new equilibrium position $\boldsymbol{X}_{0}$ (see Table 1). Indeed, we see decrease

Table 1. Eigenvectors $\xi_{j}(j=1 . .8)$, which form the basis of eightdimensional space of atomic displacements for the chain of $N=8$ atoms $(a=0.071 ; b=0.494 ; c=0.495 ; d=0.079 ; e=0.304 ; f=0.401$; $g=0.397 ; h=0.298)$.

\begin{tabular}{|c|c|c|c|c|c|c|c|c|c|c|}
\hline$\xi_{j}$ & $\lambda_{j}$ & Irreps & \multicolumn{8}{|c|}{ Eigenvectors } \\
\hline$\xi_{1}$ & 0 & $\Gamma 1$ & 1 & 1 & 1 & 1 & 1 & 1 & 1 & 1 \\
\hline$\xi_{2}$ & 88.4 & $\Gamma 2$ & 1 & -1 & 1 & -1 & 1 & -1 & 1 & -1 \\
\hline$\xi_{3}$ & 100.6 & $\Gamma 5$ & $\mathrm{a}$ & $-b$ & 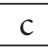 & $\mathrm{d}$ & $-\mathrm{a}$ & $\mathrm{b}$ & $-c$ & $-\mathrm{d}$ \\
\hline$\xi_{4}$ & 100.6 & & $-\mathrm{c}$ & $-\mathrm{d}$ & $\mathrm{a}$ & $-\mathrm{b}$ & $\mathrm{C}$ & $\mathrm{d}$ & $-\mathrm{a}$ & $\mathrm{b}$ \\
\hline$\xi_{5}$ & 110.6 & $\Gamma 4$ & 1 & 1 & -1 & -1 & 1 & 1 & -1 & -1 \\
\hline$\xi_{6}$ & -12.2 & $\Gamma 5$ & $-\mathrm{e}$ & $\mathrm{f}$ & $\mathrm{g}$ & $\mathrm{h}$ & $\mathrm{e}$ & $-f$ & $-g$ & $-\mathrm{h}$ \\
\hline$\xi_{7}$ & -12.2 & & $-g$ & $-\mathrm{h}$ & $-\mathrm{e}$ & $\mathrm{f}$ & $\mathrm{g}$ & $\mathrm{h}$ & $\mathrm{e}$ & $-\mathrm{f}$ \\
\hline$\xi_{8}$ & -22.2 & $\Gamma 6$ & 1 & -1 & -1 & 1 & 1 & -1 & -1 & 1 \\
\hline
\end{tabular}

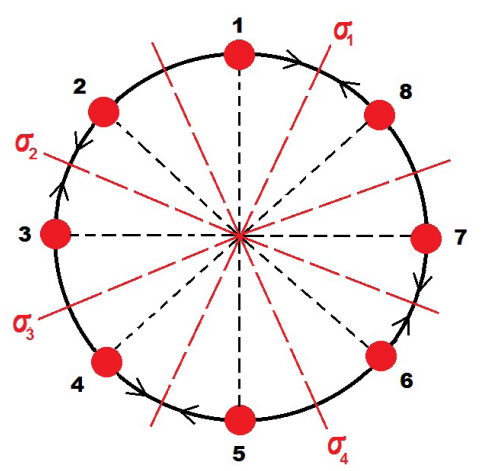

Fig. 4. Displacement pattern of the $\pi$-mode for the chain with $N=8$ particles.

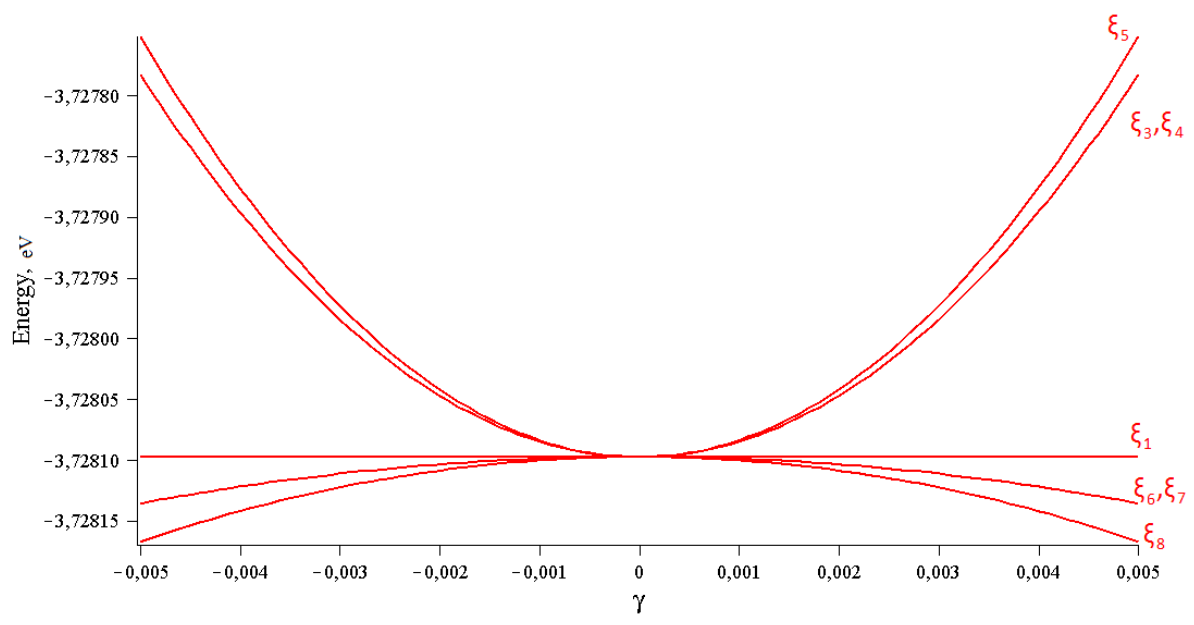

Fig. 5. Energy profiles for the L-J chain with $N=8$ particles for all basis directions $\xi_{j}(j=1 . .8)$ except $\xi_{2}$. 
of the energy for $\lambda_{j}<0\left(\lambda_{6}=\lambda_{7}, \lambda_{8}\right)$, increase of the energy for $\lambda_{j}>0\left(\lambda_{3}=\lambda_{4}, \lambda_{5}\right)$ and constant energy for $\lambda_{1}=0$.

Thus, the new EQP in carbyne with $15 \%$ strain which corresponds to the vector $\boldsymbol{X}_{0}=\left[a_{0},-a_{0}\left|a_{0},-a_{0}\right| a_{0},-a_{0} \mid a_{0},-a_{0}\right]$ with $a_{0}=0.075$ occurs to be unstable in the framework of the Lennard-Jones model. It can be proved that this conclusion is true for arbitrary number $\mathrm{N}$ of particles in the L-J chain.

\section{Study of stability of new equilibrium atomic positions in strained carbyne with the aid of DFT simulations}

The above described method can be easily applied to the study of stability properties of new equilibrium positions in strained carbyne in the framework of the ab initio DFT approach.

In Fig. 6, we present potential energy profiles calculated by ABINIT software package $[8,9]$ for $15 \%$ strained cumulene chain of $N=8$ atoms for basis directions $\xi_{j}$ from Table 1 ( $\xi_{2}$ is excluded).

The obtained results are unexpected, because the energy profiles in Fig. 6, unlike those in Fig. 5, demonstrate minima for all basis directions. Thus, the new equilibrium position, at point B in Fig. 1, being unstable in the L-J model, turns out to be stable in the framework of the DFT model. Why such discrepancy does take place? We discuss this issue in the last section of the present paper.

\section{Stability of the $\pi$-mode atomic vibrations}

Let us now consider the problem of stability of atomic vibrations near the new equilibrium positions in strained carbon chains.

In Fig. 7, we represent for $\eta=15 \%$ strain the timeevolution of the carbon atom oscillations in the right potential well (Fig. 1) approximately at the middle of its depth. The dotted line corresponds to the DFT model, while the dashed line demonstrates oscillations for the L-J model.

To analyze the stability of these oscillations we add to the initial $\pi$-mode profile

$$
\begin{gathered}
X(0)=[a(0),-a(0)|a(0),-a(0)| a(0),-a(0) \mid a(0),-a(0)], \\
a(0)=0.075 .
\end{gathered}
$$

a small perturbation in the form $\gamma \xi_{8}$ with $\gamma=10^{-3}$, where $\xi_{8}$ is the basis vector from Table 1, which corresponds to the negative eigenvalue with maximal absolute value $\left(\lambda_{8}=-22.2\right)$. It is obvious from Fig. 7 that the solution of the Newton ordinary differential equations, corresponding to the LJ model, demonstrates hard instability (dashed line) already at ten oscillation periods $(t \approx 10 T)$. In contrast to this result, one can see from Fig. 7 that the DFT solution for the same initial conditions (dotted line) demonstrates a fine stability, at least up to time interval $t \approx 100 T$. The small modulation of

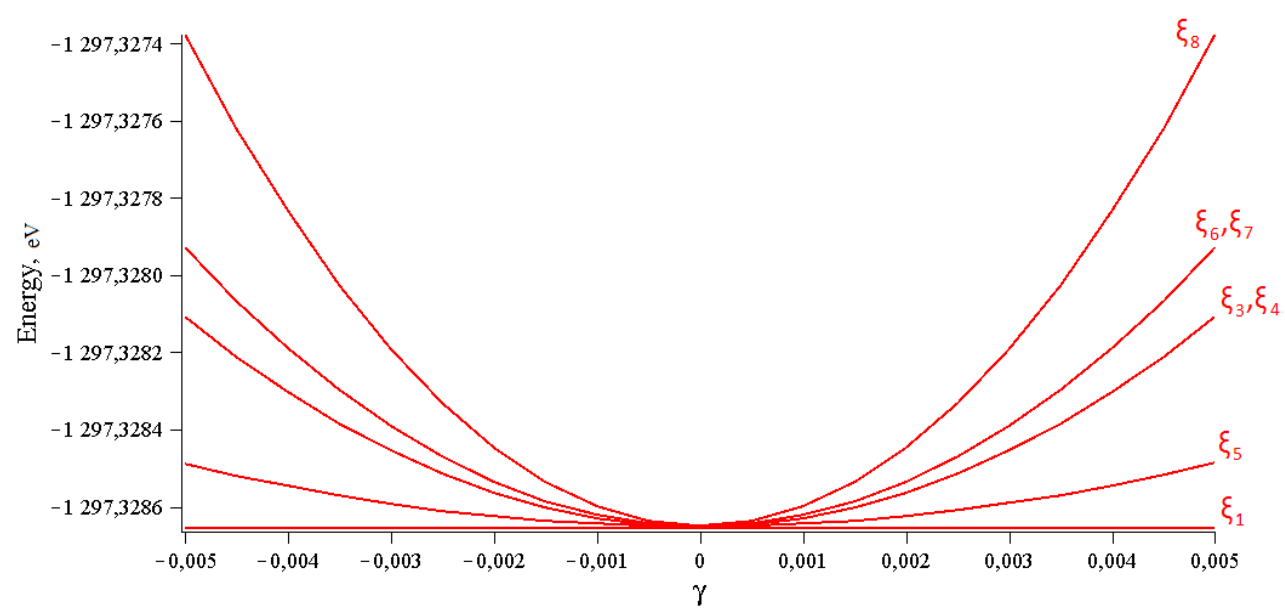

Fig. 6. Energy profiles for carbyne chain of $N=8$ particles for basis directions $\xi_{j}$ which are obtained in the framework of ab initio DFT model.

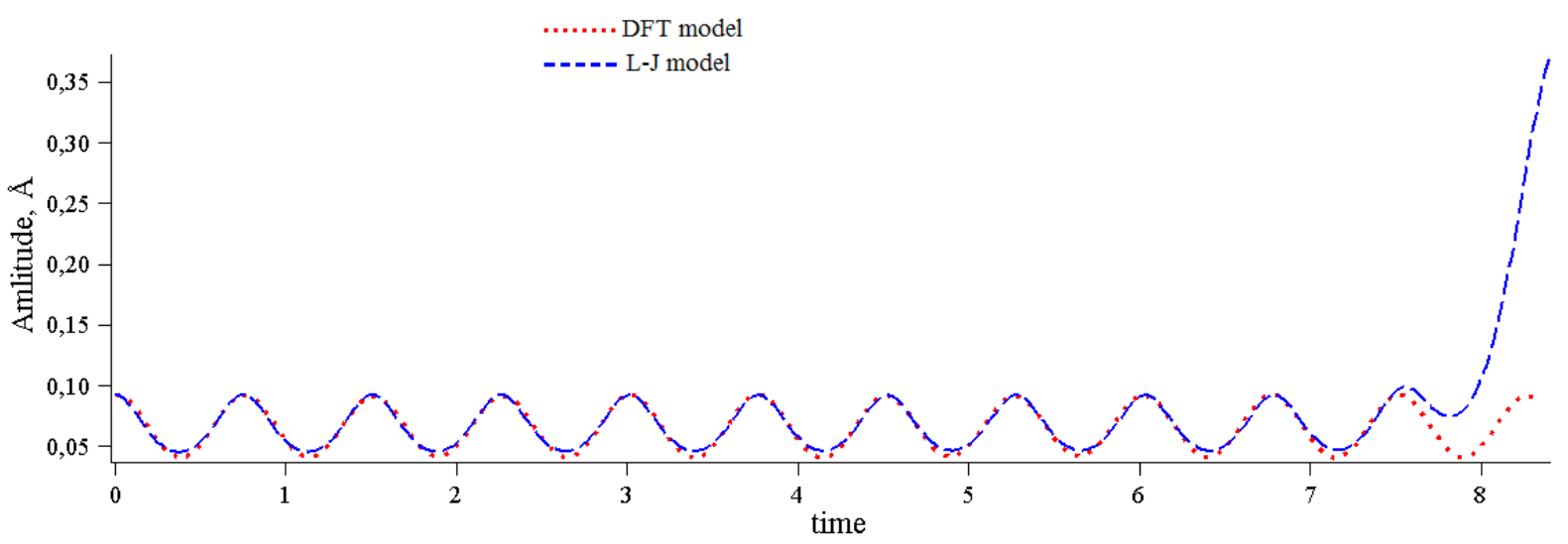

Fig. 7. Time-evolution of $\pi$-mode initial profile (4) in DFT and L-J models for eight-particle carbyne chain under $15 \%$ strain. 
the periodic oscillations in the DFT model is induced by the above-mentioned perturbation $\gamma \xi_{8}\left(\gamma=10^{-3}\right)$ artificially added to the exactly periodic initial profile (4).

Thus, we can conclude that $\pi$-mode atomic vibrations in the L-J model are unstable, while they demonstrate stability in the DFT model (at least, by visual inspection). Remember, that the analogical discrepancy between static properties of the L-J model and the DFT model was discussed in the previous section.

Because of importance of this conclusion, we verify by the rigorous Floquet method that periodic atomic vibrations in the L-J chain are indeed unstable. The similar stability analysis for the DFT model was not carried out yet because of some computational difficulties.

\section{Discussion and conclusion}

The present work is a continuation of our previous paper [6]. Both of these studies are devoted to analysis of longitudinal nonlinear atomic vibrations in strained carbon chains in the framework of ab initio DFT approach. Let us summarize the obtained results.

In [6], we have revealed that under sufficiently large uniform strain $(\eta>11.2 \%)$ radical transformation of the cumulene structure takes place as the result of condensation of the $\pi$-mode, which becomes a soft mode in a certain range of its amplitude. Indeed, in this case, the old equilibrium positions, around which carbon atoms vibrate in the case of the small strain, lose their stability and two new EQPs arise near each of them. This transformation corresponds to the Peierls phase transition in onedimensional crystal and is associated with doubling of the unit cell. As a result, carbyne chain transforms from the cumulene to polyyne form. This phase transition in strained carbyne chains was discovered in the work [1], which was also carried out by ab initio DFT simulations, but from another positions compared to those of [6]. Indeed, we have studied nonlinear vibrations in carbyne chains, while the authors of [1] explored their static structure.

As was emphasized in [1], the above phase transition is a phenomenon of great physical importance because it leads to a qualitative change in the electron spectrum of cumulene. Indeed, as a result of appearance in this spectrum of energy gap, conductive cumulene transforms into polyyne which is insulator or semiconductor. In turn, it is expected that such change in electrical properties under mechanical strain can be used in some nanodevices.

In [1], a simple quantum-mechanical model of one particle moving in double-well potential was introduced to explain the properties of the Peierls phase transition found in the framework of DFT approach. Unlike this, in [6], for the same purpose, a simpler classical model was introduced, which represents the chain of mass-points whose interactions are described by the Lennard-Jones potential (L-J chain). Using this model, we were able to explain not only the static properties, such as appearance of new EQPs, etc., but also the properties of nonlinear vibrations near these new equilibrium positions. Moreover, the approach developed in [6] allows us to consider the condensation of two other symmetry-determined Rosenberg nonlinear normal modes discussed in [6] (besides the $\pi$-mode), which correspond to multiplication of vibrational unit cell three- or four-times compared to that of the cumulene equilibrium state. In turn, such approach leads to a prediction of possibility for existence of two new forms of carbyne with the bond length alternations different from that of polyyne (these results will be published elsewhere).

Let us note that all types of symmetry-determined periodic and quasiperiodic vibrations in physical systems with discrete symmetries can be obtained with the aid of specific grouptheoretical methods developed in the theory of bushes of nonlinear normal modes [12]. The application of this theory to various mechanical systems can be found in [11-19].

Now let us return to consideration of stability properties of the new EQPs and of the periodic oscillations in their vicinity, which are discussed in Sections 3 and 4 of this paper. We present there computational experiments demonstrating that our Lennard-Jones model, which is rather accurate for prediction of new static and dynamical properties of the strained carbon chains, turns out to be fully unsatisfactory for analyzing stability of new atomic equilibrium positions and stability of oscillations around them. Now we can indicate the following causes of such phenomenon.

As was already emphasized in Sec. 2, unlike the L-J model, the DFT model is more adequate because it takes into account electron shells of each carbon atom, which adapt to any change in nuclear configuration, while the L-J model deals with bare mass points. In other words, many degrees of freedom correspond to each site in the DFT model, while only one variable is associated with every site in the Lennard-Jones model. There is also a mathematical cause of the above discrepancy. Indeed, in the case of the L-J model, we study the stability of a solution of ordinary differential equations (classical Newton equations), while in the case of the DFT model one must analyze the stability of a system of cumbersome integro-differential equations (Kohn-Sham quantum-mechanical equations).

Thus, the L-J model describes sufficiently well the existence of new equilibrium positions in strained carbyne, but it turns out to be unsatisfactory for analysis of their stability. We feel that such discrepancy between the results obtained by molecular dynamics methods and those by DFTsimulations may be typical for different physical problems.

Aknowledgements. The authors are sincerely grateful to Profs. V. P. Sakhnenko, S. V. Dmitriev and N. V. Ter-Oganessian for useful discussions, and to I. P. Lobzenko for assistance in application of the software package ABINIT. This work was supported by the Russian Science Foundation (Grant No. 1413-00982).

\section{References}

1. V. I. Artyukhov, M. Liu, and B. I. Yakobson, Nano Lett. 14, 4224 (2014).

2. G. Casillas, A. Mayoral, M. Liu, V. I. Artyukhov, A. Poncea, B. I. Yakobson, M. J. Yacaman, Carbon 66, 436 (2014).

3. M. Liu, V.I. Artyukhov, H. Lee, F. Xu, B. I. Yakobson, ACS Nano 7, 10075 (2013).

4. P. B. Sorokin, H. K. Lee, L. Yu. Antipina, A. K. Singh, B. I. Yakobson, Nano Lett. 11, 2660 (2011). 
5. S. Cahangirov, M. Topsakal, S. Ciraci. Phys. Rev. B82 (19), 195444 (2010).

6. G. M. Chechin, D. A. Sizintsev, O. A. Usoltsev, Letters on materials 6 (2), 146 (2016).

7. W. Cochran, Phys. Rev. Lett. 3 (9), 412 (1959).

8. X. Gonze et al. Comput. Phys. Commun. 180, 2582 (2009).

9. www.abinit.org.

10. W. Kohn. Rev. Mod. Phys. 71, 1253 (1999).

11. G. M. Chechin, D. S. Ryabov, K. G. Zhukov, Physica D203, 121 (2005).

12. G. M. Chechin, V.P. Sakhnenko, Physica D117, 43 (1998).

13. G.M. Chechin, A.V. Gnezdilov, M.Yu. Zekhtser,
Int. J. Nonlinear Mech. 38, 1451 (2003).

14. G. M. Chechin, K.G. Zhukov, Phys. Rev. E73., 36216 (2006).

15. G. M. Chechin, D.S. Ryabov, Phys. Rev. E69, 036202 (2004).

16. G.M. Chechin, S.A. Shcherbinin, Commun. Nonlinear Sci. Numer. Simulat. 22, 244 (2015).

17. G.M. Chechin, D.S. Ryabov, S.A. Shcherbinin, Phys. Rev. E92, 012907 (2015).

18. G.S. Bezuglova, G.M. Chechin, P.P. Goncharov, Phys. Rev. E84, 036606 (2011).

19. G. M. Chechin, D. S. Ryabov, S. A. Shcherbinin, Letters on materials 6(1), 9 (2016). 